DOI: 10.2478/adms-2014-0021

\author{
G. Varughese $^{1}$, V. Rini ${ }^{1}$, S.P. Suraj ${ }^{1}$ K.T. Usha ${ }^{2}$ \\ ${ }^{1}$ Department of Physics, Catholicate College, Pathanamthitta, Kerala, India -689645 \\ ${ }^{2}$ Department of Chemistry, St. Cyril's College, Adoor, Kerala \\ gvushakoppara@yahoo.co.in
}

\title{
CHARACTERISATION AND OPTICAL STUDIES OF COPPER OXIDE NANOSTRUCTURES DOPED WITH LANTHANUM IONS
}

\begin{abstract}
Copper Oxide is an extensively studied group II-VI semiconductor with optical properties. It exhibits a wide variety of morphologies in the nano regime that can be grown by tuning the growth habit of the CuO crystal. $\mathrm{CuO}$ nano materials with an average particle size of $15-27 \mathrm{~nm}$ are synthesized by chemical route. XRD, SEM, FTIR UV-Vis and EDS characterize the samples. The percentage of doping material is confirmed from the EDS spectra. The average crystal size of the prepared $\mathrm{CuO}$ : La nanopowder is determined by XRD. The UV absorption spectra revealed the absorption edge at wavelength $389 \mathrm{~nm}$ indicating the smaller size of CuO:La nano particle. The optical direct band gap energy of doped $\mathrm{CuO}$ nanoparticle is found to be in the range 3.149 $\mathrm{eV}$. The increasing red shift with decreasing particle size suggests that the defects responsible for the intra gap states are primarily surface defect. The La doped $\mathrm{CuO}$ is highly effective and can significantly enhance the photo catalytic degradation.
\end{abstract}

Key words: Nanomaterial, Doping, Optical properties

\section{INTRODUCTION}

Nanomaterials are different from bulk materials and isolated molecules because of their unique optical, electronic and chemical properties $[1,2]$. They manifest extremely fascinating and useful properties, which can be exploited for a variety of structural and non-structural applications [3]. Metal oxide nanoparticles belong to a family of nanomaterials that have been manufactured on a large scale for both industrial and household applications, and they hold promise for future applications [4, 5]. Copper Oxide is an extensively studied group II-VI semiconductor with optical properties that permits stable emission at room temperature having immense application in sensors, field emission and photonic devices [6]. Nanoparticles of $\mathrm{CuO}$ can be used as gas sensors, optical switch, and magnetic storage media owing to its photoconductive and photochemical properties [7]. Furthermore it is a promising semiconductor for solar cell fabrication due to its suitable optical properties. $\mathrm{CuO}$ 
nanoparticles have immense medical applications [8, 9, and 10]. It possesses useful photovoltaic and photoconductive properties because $\mathrm{CuO}$ crystal structures have a narrow band gap $[11,12]$. $\mathrm{CuO}$ nanoparticles have been applied in different areas, including sensors catalysis, batteries, high temperature superconductors, solar energy conversion, and field emission emitters [13]. Ohwada $\mathrm{K}$ et al. [14] carried out normal coordinate analysis of the optically active vibrations of doped lanthanum copper oxide with the assumption of an infinite $\left[\left(\mathrm{CuO}_{2}\right) \mathrm{O}_{2}\right]_{6}$ - layer model. With respect to the results of this analysis, some of the observed bands have been successfully assigned to the $\mathrm{Cu}-\mathrm{O}$ lattice vibrations. In addition, the force constants concerning $\mathrm{Cu}-\mathrm{O}$ bonds have also been obtained within the framework of a modified valence force field. It was found that this analysis based on the infinite layer model is useful for assigning the relatively high vibrational frequencies observed at the centre of the first Brillouin zone in doped lanthanum copper oxide. Golosovsky IV et al.[15]demonstrated a noncollinear anti-ferromagnetic structure with the transition temperature of approximately $130 \mathrm{~K}$ and propagation vector $\mathrm{k}=\left[\begin{array}{lll}0 & 1 / 2 & 0\end{array}\right]$ in the monoclinic compound $\mathrm{La}_{2} \mathrm{Cu}_{2} \mathrm{O}_{5}$ by using single-crystal neutron diffraction method. They obtained the values and directions of the magnetic moments and crystal structure parameters by least-squares refinement. The results showed that $\mathrm{La}_{2} \mathrm{Cu}_{2} \mathrm{O}_{5}$ possess low-dimensional magnetic behavior with critical index beta $=$ $0.228(8)$, like rare-earth cuprates $\mathrm{R}_{2} \mathrm{CuO}_{4} \cdot \mathrm{La}_{2} \mathrm{O}_{3}$ is an ingredient for the manufacture of piezoelectric and thermoelectric material. In is used to increase piezoelectric coefficient and improve product energy conversion efficiency and catalytic applications. Lanthanum is used to improve burning rate of propellant of aircraft. It is a laser material used in X-ray imaging intensifying screens, phosphors as well as in ceramics. Copper oxide nanoparticle has been doped with Lanthanum and studied the effect on energy band gap on doped material [16]. The doping of $\mathrm{La}$ ions may enhance optical properties of $\mathrm{CuO}$ nano particles. No study on the optical properties of $\mathrm{CuO}$ : $\mathrm{La}$ was reported so far. Hence the effect of doping $\mathrm{La}$ with $\mathrm{CuO}$ nanoparticles was studied in this paper.

\section{EXPERIMENTAL}

\section{Synthesis and characterization}

$\mathrm{CuO}$ nanoparticles are prepared by Chemical precipitation route. $\mathrm{CuO}$ nanoparticles are prepared from copper nitrate solution and ammonia. In this method one molar solution of $\mathrm{Cu}\left(\mathrm{NO}_{3}\right)_{2}$ is prepared. For this, 24.16gm of copper nitrate is taken and dissolved in $100 \mathrm{ml}$ distilled water. Mix $25 \mathrm{ml}$ ammonia solution in $75 \mathrm{ml}$ water. These two solutions are kept in two burettes and drop simultaneously into a conical flask containing water and stirred. O.1M Lanthanum dioxide solutions is dropped along with other two solutions. A precipitate is formed which is washed off using distilled water and acetone. In order to prevent agglomeration 4-5 drops of Tri Ethyl Amine (TEA) was added to solution. After proper centrifugation the residue is dried at $80^{\circ} \mathrm{c}$ for one hour in the temperature controlled furnace. Annealing at $250^{\circ} \mathrm{C}$ for two hours gives copper oxide. When colour of the sample becomes coffee brown $\mathrm{CuO}$ doped with Lanthanum ions is formed. 
The orientation and crystallinity of the powder were studied using Rigaku DMAX diffractometer using $\mathrm{Cu}-\mathrm{K} \alpha$ radiation monochromatised with a graphite crystal and high temperature attachment in $\theta-2 \theta$ geometry. The surface topography and microstructure were studied using Field Emission Scanning Electron Microscopy (FESEM). Perker Elmer Lamda $25 \mathrm{UV}$-Vis spectrometer was used to study the optical properties of nanopowder. FTIR spectroscopy uses Michelson interferometer to produce an interferogram. Energy Dispersive $\mathrm{X}$-ray Spectrum Analysis (EDAX) was used to determine percentage composition of La in $\mathrm{CuO}$.

\section{RESULTS AND DISCUSSIONS}

\section{Characterization using XRD}

The XRD pattern, Figure 1(a-c), consists of sharp intense peaks of CuO:La which confirms the good crystalline nature of $\mathrm{CuO}$ and peaks originated from(100),(002),(101),(102), (110),(103),(112),(201),(200) and (202) reflections of hexagonal $\mathrm{CuO}$ [5]. The XRD techniques are widely used for the particle size and structure determination of nanoparticles. The patterns are compared with JCPDS file No: 80-0075 comparing the observed data with the JCPDS file. The percentage variation of hkl values are shown below in the Table 1.From the table it is noted that percentage variation of hkl values very small, which indicates the change in monoclinic structure of the $\mathrm{CuO}$ nanoparticle with doping of La ions is insignificant.

Table 1. Comparison of the observed data with the JCPDS file of Copper Oxide. Percentage Variation of $d$ values of nanocrystalline CuO:La annealed at $250^{\circ} \mathrm{C}$

\begin{tabular}{|c|l|l|l|l|l|}
\hline S1 No & $\begin{array}{c}\text { Observed } \\
\text { Value of } \\
\text { CuO:La } \\
\text { nanoparticle }\end{array}$ & $\begin{array}{c}\text { Standard d- } \\
\text { value of CuO } \\
\text { nanoparticle }\end{array}$ & h k l & $2 \theta$ & $\begin{array}{c}\text { \% variation } \\
(\times 10-3)\end{array}$ \\
\hline 1 & 3.44106 & 3.44400 & 111 & 25.871 & 0.853 \\
2 & 2.78219 & 2.72300 & 400 & 32.147 & 0.0217 \\
3 & 2.68859 & 2.63500 & 410 & 33.685 & 0.0203 \\
4 & 2.45244 & 2.42900 & 420 & 36.616 & 9.567 \\
5 & 2.14768 & 2.13300 & 331 & 42.037 & 6.882 \\
6 & 1.77636 & 1.7880 & 610 & 51.397 & 6.510 \\
7 & 1.70950 & 1.72500 & 441 & 56.564 & 6.682 \\
8 & 1.5834 & 1.57300 & 402 & 58.22 & 6.6115 \\
\hline
\end{tabular}




\section{Determination of particle Size from XRD Pattern of CuO:La}
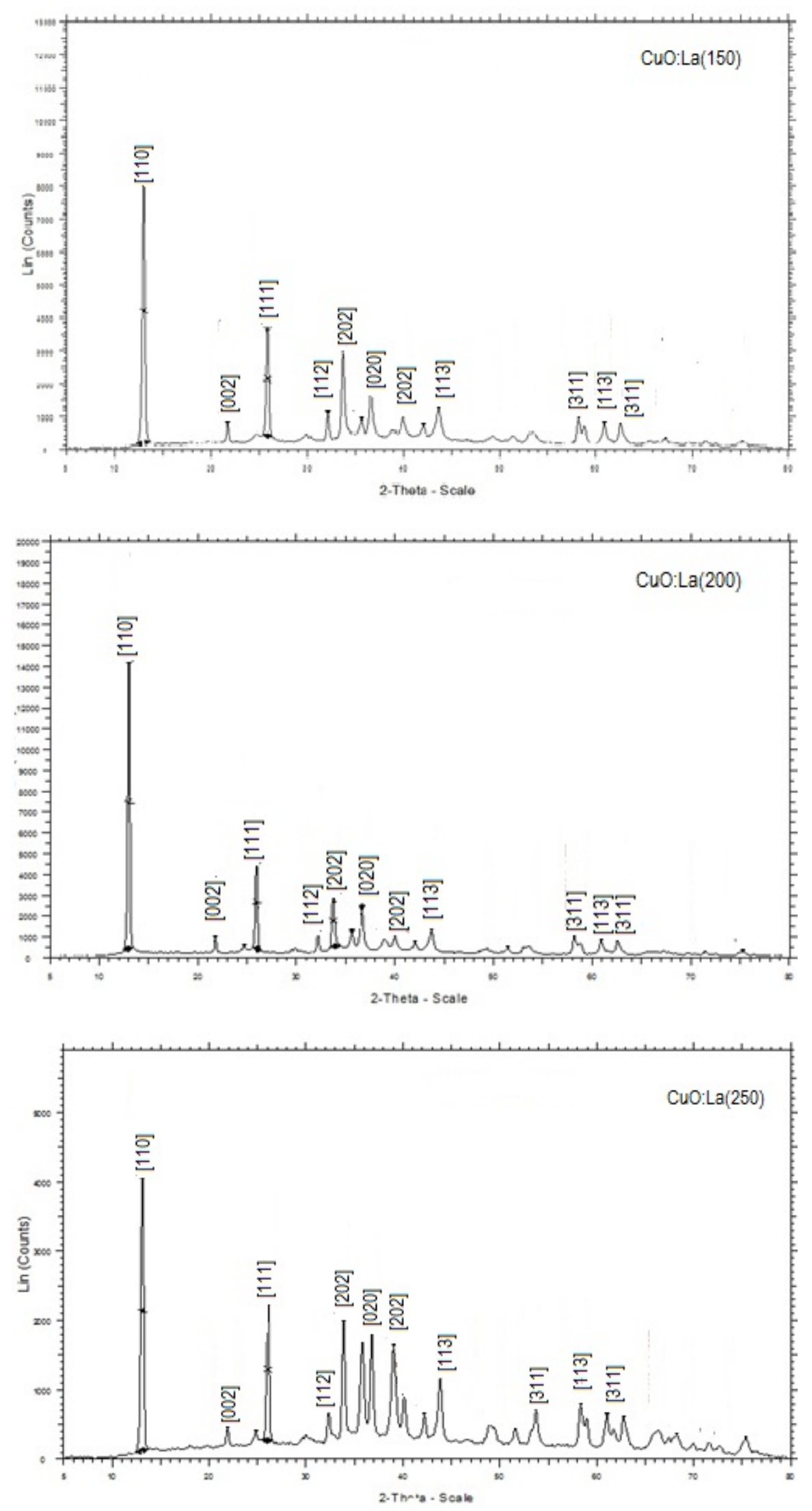

Fig. 1 (a-c). XRD pattern of CuO:La at $150,200,250^{\circ} \mathrm{C}$ 
The degree of crystallinity of nanoparticles increases with annealing temperature. The percentage of lattice contraction with annealing temperature can also be studied using X-ray diffraction pattern. Particle Size, can be calculated by the formula [9]

Debye- Scherrer's formula $D=K \lambda / \beta \operatorname{Cos} \theta$.

$\mathrm{K}=0.89, \lambda$ the $\mathrm{X}$-ray wavelength $=0.154095 \mathrm{~nm}, \beta$ the full wavelength at half maximum and $\theta$ the half diffraction angle. The crystal size of $\mathrm{CuO}$ : La nano particle at $150,200,250^{\circ} \mathrm{C}$ was calculated from FWHM and tabulated in Table 2.

Table: 2. Particle size measurements of CuO:La from XRD data

\begin{tabular}{|c|c|c|c|c|c|}
\hline $\begin{array}{c}\text { Temp } \\
\left({ }^{\circ} \mathrm{C}\right)\end{array}$ & FWHM & $\beta \times 10-3$ & $2 \theta$ & $\theta$ & $\begin{array}{c}\text { Particle size } \\
(\mathrm{D}) \mathrm{Nm}\end{array}$ \\
\hline 150 & 0.343 & 5.98 & 13.35 & 6.535 & 23 \\
\hline 200 & 0.295 & 5.14 & 12.892 & 6.446 & 27 \\
\hline 250 & 0.24 & 4.18 & 12.948 & 6.474 & 33 \\
\hline
\end{tabular}

\section{Variation of Particle size of CuO:La Nanoparticle with temperature}

It was found that the crystallite size of $\mathrm{CuO}$ : La nanopowder was $23 \mathrm{~nm}$ at $150^{\circ} \mathrm{C}$ which increased to $27 \mathrm{~nm}$ at $200^{\circ} \mathrm{C}$ and $33 \mathrm{~nm}$ at $250^{\circ} \mathrm{C}$. From the figure it is clear that the intensity of crystalline peaks increases with increase in temperature. Simultaneously the peaks become narrower as the temperature was increased showing the increase in crystallite size The continuous increase in the particle size with temperature can be attribute to the atomic diffusion. From the atomic perspective, diffusion is a stepwise migration of atom from lattice site to lattice site. In fact, the atoms in solid material are in constant motion, rapidly changing position. For an atom to make such a move the atom must have sufficient energies to break bonds with its neighbor atoms and then cause some lattice distortion during the displacement. As the temperature increases the atom gain sufficient energy for diffusion motion and thereby increasing crystallite size [18]. According to Ostwald ripening phenomena the increase in the particle size is due to the merging of the smaller particles into larger ones as a result of potential energy difference between small and large particles and can occur through solidstate diffusion [17].

\section{Scanning Electron Microscopy (SEM) of CuO:La}

The Scanning Electron Micrographs of CuO: La, Fig.2, nanomaterials synthesized under aqueous medium. The SEM image of $\mathrm{CuO}$ : La nanoparticle under high magnification is shown below. Spherical shaped morphology is observed in the micrograph of $\mathrm{CuO}$ : La, Fig. 2. The SEM pictures show distinguished spherical morphology with self aligned prismatic nanoparticles. The morphology of $\mathrm{CuO}$ nanopowder as revealed by FESEM showed nanoparticle of size $15-100 \mathrm{~nm}$. 


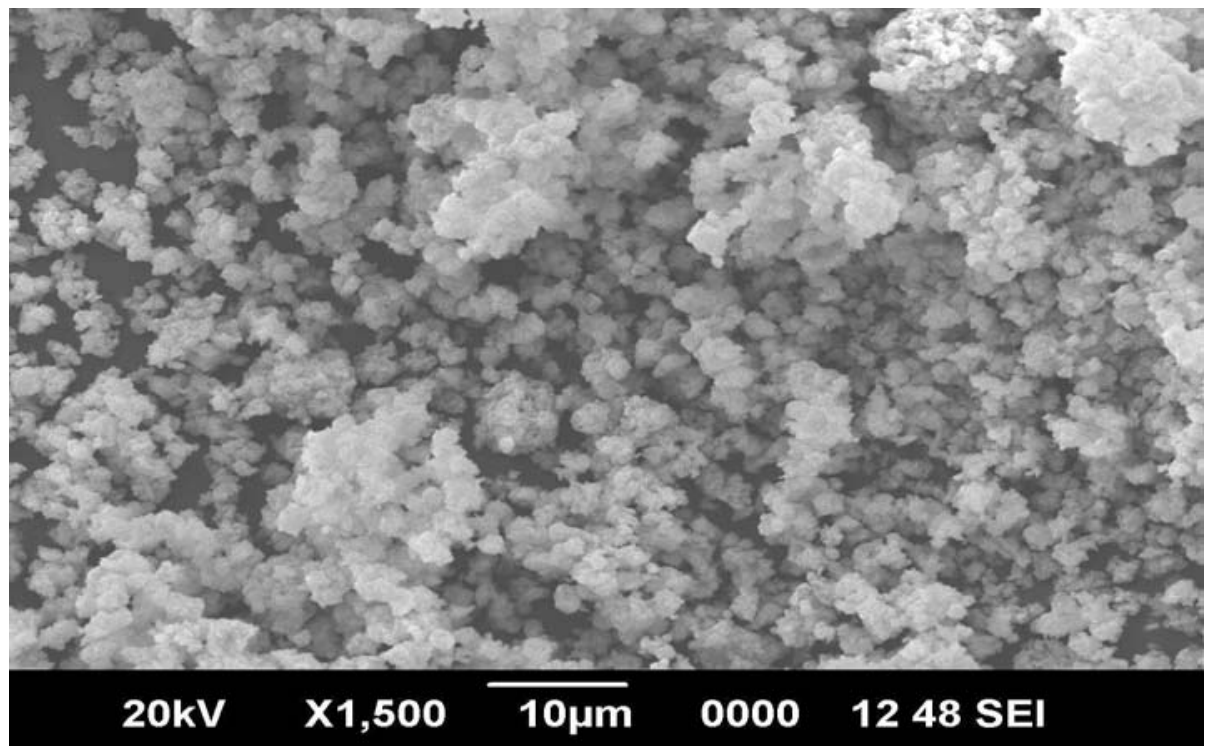

Fig. 2. SEM Image of CUO:La nanoparticle under high magnification

\section{Energy Dispersive Spectrum Analysis (EDS) of CuO: La nanoparticles}

EDAX spectrum, Figure 3, plot not only identifies the elements corresponding to each of its peaks, but the type of X-ray to which it corresponds as well. The higher a peak in a spectrum, the more concentrated the element is in the spectrum. Spherical shaped morphology is observed in the micrograph of $\mathrm{CuO}: \mathrm{La}$.

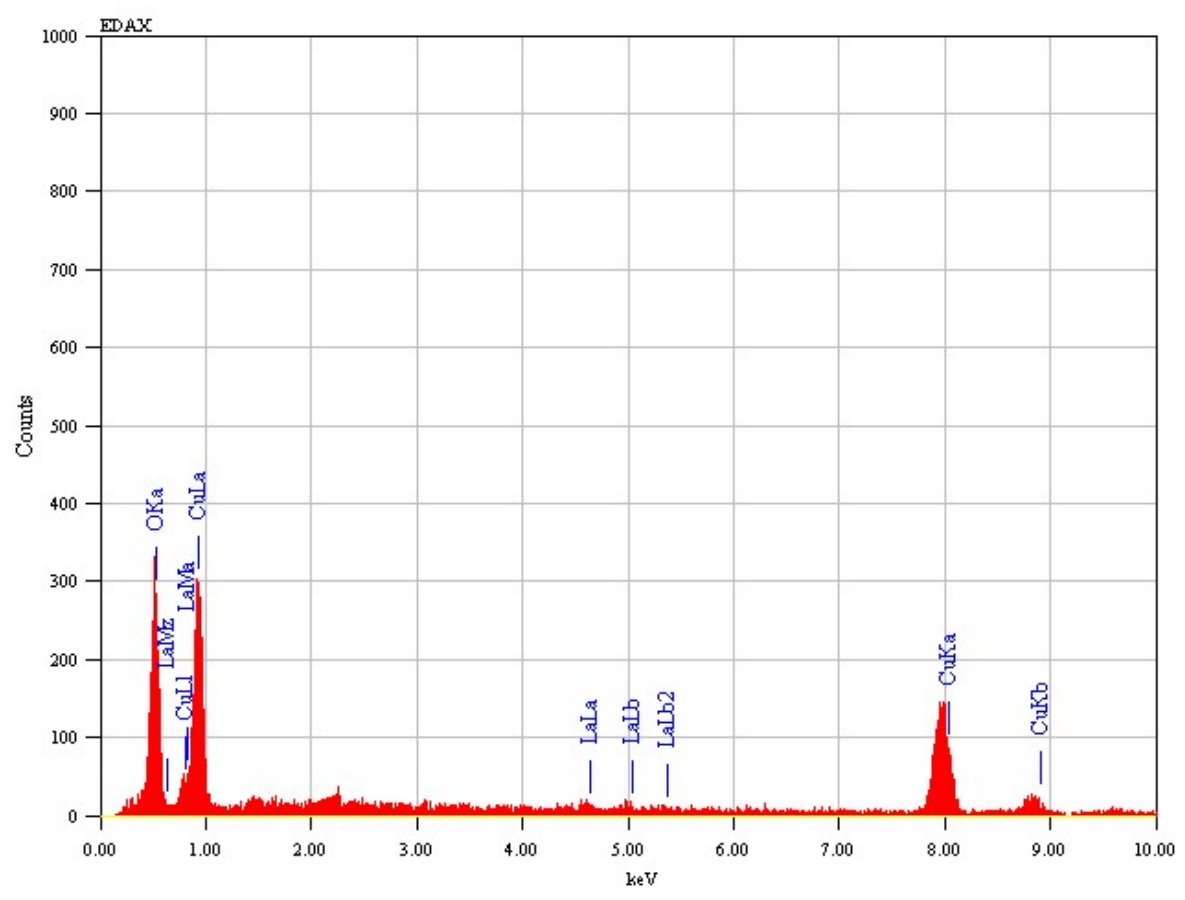

Fig 3. EDAX of CuO: La nanoparticle

The dried powder of the sample was analyzed on Energy Dispersive X-ray Analysis (EDAX or EDS) technique. The peaks have confirmed the presence of Copper, Oxygen and Lanthanum atoms. The average atomic weight percentage ratio of $\mathrm{Cu}, \mathrm{O}$, and $\mathrm{La}$ in $\mathrm{CuO}: \mathrm{La}$ 
nanoparticle was 31.17: 67.01: 4.648. The energy ratio (in $\mathrm{keV}$ ) of the elements were 31.37: 0.525: 4.68. The data's are presented in the Table 4. The presence of doped rare earth element Lanthanum ions in $\mathrm{CuO}$ nanopowder was confirmed by the analysis.

Table 4. Composition of elements in CuO:La sample

\begin{tabular}{llll}
\hline Sample & Element & $\begin{array}{l}\text { Atomic } \\
\text { percentage }\end{array}$ & $\begin{array}{l}\text { Energy } \\
(\mathrm{keV})\end{array}$ \\
\hline & $\mathrm{Cu}$ & 31.17 & 31.37 \\
$\mathrm{CuO}: \mathrm{La}$ & $\mathrm{O}$ & 67.01 & 0.525 \\
& $\mathrm{La}$ & 4.68 & 4.68 \\
\hline
\end{tabular}

\section{FTIR Spectrum of CuO: La}

FTIR spectroscopy uses Michelson interferometer to produce an interferogram and the spectrum of $\mathrm{CuO}$ : La is shown in the Fig. 4. It has been shown that as particle size decreases, increase in frequency for the bond (blue shift) is observed in nanoparticles .Bands at 416.35 $\mathrm{cm}^{-1}$ and $599.23 \mathrm{~cm}-1$ are assigned to the stretching vibrations of $\mathrm{Cu}-\mathrm{O}$. The stretching frequency of bulk $\mathrm{CuO}$ is $424 \mathrm{~cm}^{-1}$. Here a blue shift is observed in that frequency i.e., that frequency due to quantum confinement Three intense bands were centered at $1384.34 \mathrm{~cm}^{-1}$, $1041.54 \mathrm{~cm}^{-1}$ and $1556.58 \mathrm{~cm}^{-1}$ and are attributed to the stretching vibrations of $\mathrm{C}=\mathrm{O}, \mathrm{C}=$ $\mathrm{C}$ and $\mathrm{C}-\mathrm{H}$ groups in acetate species, which suggests its presents as absorbed species in the surface of nanoparticles. The broad absorption peak centered at $3423.61 \mathrm{~cm}^{-1}$ and $1626.40 \mathrm{~cm}^{-}$ ${ }^{1}$ corresponds to $\mathrm{O}-\mathrm{H}$ stretching and bending frequencies of $\mathrm{H}_{2} \mathrm{O}$, indicating the existence of water in the surface of nanoparticles [19].Variations of the peak positions of $\mathrm{CuO}$ :La relative to undoped $\mathrm{CuO}[27,28]$ are presented in the Table 3. From the data's presented in the table revealed that the doping with lanthanum ions induces a shift in the absorption bands of the undoped $\mathrm{CuO}$.

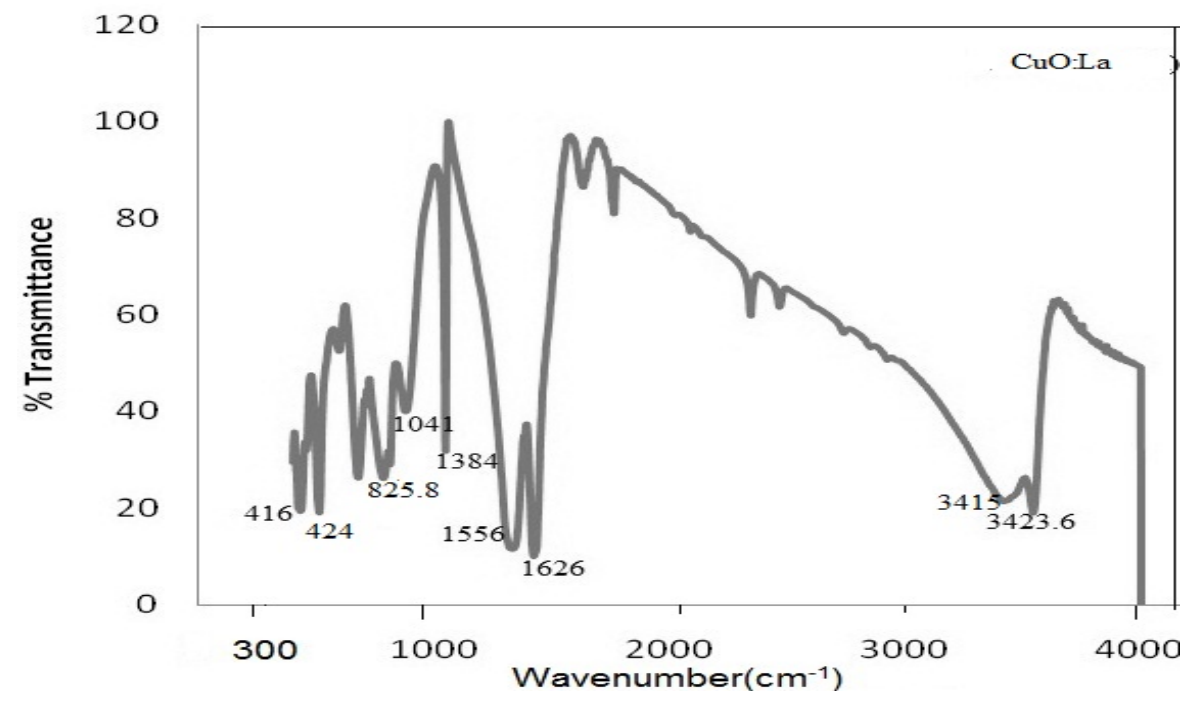

Fig. 4. FTIR Spectra of nanocrystalline CuO:La 
The peak observed at $825.80 \mathrm{~cm}^{-1}$ may be the presence of some La in the Copper Oxide nanoparticle. The broad absorption peak centered at $3415.73 \mathrm{~cm}-1$ corresponds to $\mathrm{O}-\mathrm{H}$ stretching of water indicating the existence of water in the surface of nanoparticles [20

Table 3. Comparison of Vibrational modes of undoped and La doped CuO nanoparticle

\begin{tabular}{lll}
\hline $\mathbf{C u O}\left(\mathbf{c m}^{-1}\right)$ & CuO:La ( cm-1) & Vibrational modes \\
\hline 3573 & 3423.61 & $\mathrm{OH}$ \\
3486 & 3415.73 & $\begin{array}{l}\mathrm{N}-\mathrm{H} \text { vibration of amine } \\
\text { group }\end{array}$ \\
2361 & 2822 & $\mathrm{O}=\mathrm{C}=\mathrm{O}$ vibration \\
1319 & 1384.34 & $\mathrm{C}=\mathrm{O}$ Stretching \\
1112 & 1041.54 & $\mathrm{C}=\mathrm{C}$ stretching \\
1634 & 1626.4 & OH deformation \\
984 & 1556.58 & $\mathrm{C}-\mathrm{H}$ \\
606 & 825.8 & La-O stretching vibrations \\
432 & 599.23 & Stretching of CuO \\
426 & 424 & Stretching of CuO \\
\hline
\end{tabular}

\section{UV Absorption and Reflection Spectra}

The optical absorption spectrum [21] was used to study the optical properties of the synthesized $\mathrm{CuO}$ nanoparticles; from this the band gap and the type of electronic transitions were determined. When a semiconductor absorbs photons of energy larger than the gap of the semiconductor, an electron is transferred from the valence band to the conduction band there occurs an abrupt increase in the absorbency of the material to the wavelength corresponding to the band gap energy. The relation of the absorption coefficient $(\alpha)$ to the incidental photon energy depends on the type of electronic transitions. When in this transition, the electron momentum is conserved, the transition is direct, but if the momentum does not conserve this transition it must be attended by a photon this is an indirect electronic transition [21]. Energy band gap studies of these materials have been reported using absorption spectra. The fig.5 depicts the optical absorption spectrum of $\mathrm{CuO}: \mathrm{La}$ nanopowder prepared under aqueous conditions. The UV visible spectra displayed excitonic absorption peak at $280 \mathrm{~nm}$. It shows that the absorption edge at $389 \mathrm{~nm}$. The material is reported as direct band gap material. For higher values of absorption coefficient, optical absorption shows a power load dependence on photon energy. 
A. Determination of Band gap energy from UV absorption Spectra.

Energy band gap studies of these materials have been reported using absorption spectra, Figure 6 . The material is reported as direct band gap material. For higher values of absorption coefficient, optical absorption shows a power load dependence on photon energy [21].

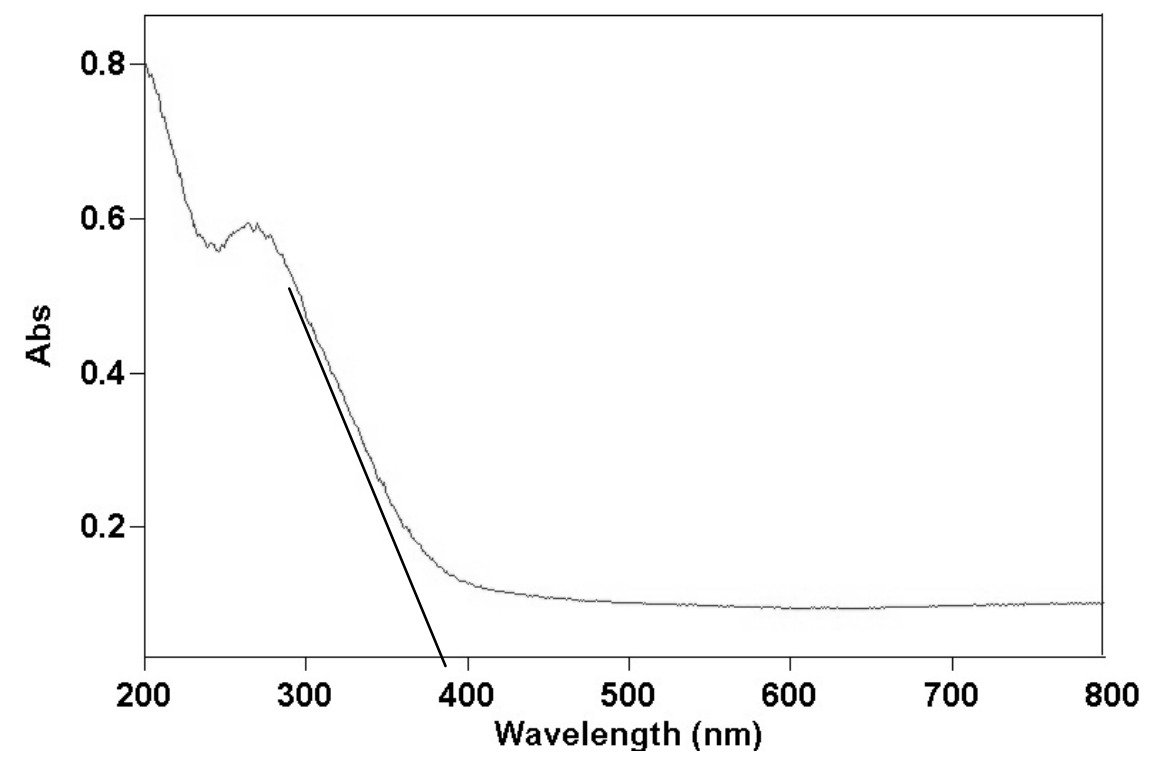

Fig. 5. UV Absorbtion spectra of CuO: La nanoparticle

$$
E_{g}=h v_{g}=h c / \lambda_{g}
$$

$E_{g}$ is the optical band gap. Optical energy gap is obtained by extrapolating the linear portion of the absorption spectrum to zero. The energy band gap for $\mathrm{La}$ doped $\mathrm{CuO}$ is calculated using $\lambda_{\mathrm{g}}=389 \mathrm{~nm}$ as $3.149 \mathrm{eV}$. But the standard band gap energy for $\mathrm{CuO}$ nanoparticle is $4.68 \mathrm{eV}$ [23]. On doping band gap energy decreases. Optical absorption shows that the direct band gap verses indirect band gap permits the determination of the crystallinity of a material. Energy band gap studies [22] of these materials have been reported using absorption spectra. The red shift in the band gap energy and reduced electron hole recombination rate make the product material an ideal photo catalyst to harvest solar radiation for various applications. With decreasing particle size, a strong hybridization of the s-p states of the $\mathrm{CuO}$ host and the $\mathrm{d}$ states of the La impurity is likely to occur [22]. The increasing red shift with decreasing particle size suggests that the defects responsible for the intra gap states are primarily surface defect [25]. The La doped $\mathrm{CuO}$ is highly effective and can significantly enhance the photo catalytic degradation. Further these nanoparticles could be used in semiconductor devices such as photo amplifiers, modulators for optical fiber communication system etc. For Copper and oxygen atom paramagnetic and ferromagnetic moments increases with decreasing size. The role of oxygen vacancies is understood to relate to the generation of free carrier mediating ferromagnetism between copper spins [26]. 


\section{UV Reflectance spectra of CuO: La}

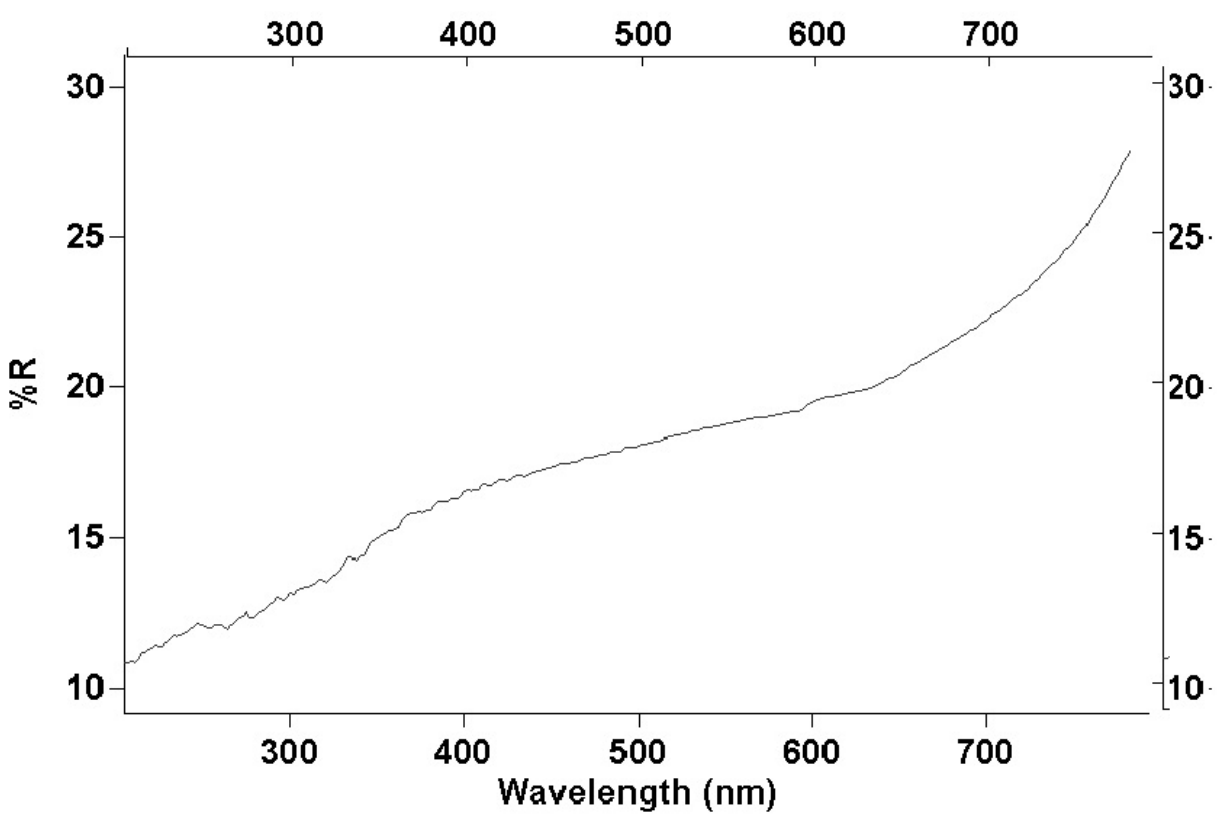

Fig. 6. UV Reflection spectra of Cu O: La Nanoparticle

The UV Reflection spectra depicted in Fig. 6 shows a gradual increase in the percentage of reflectance with wavelength.

\section{CONCLUSION}

The size and crystal structure of $\mathrm{CuO}$ doped with lanthanum was studied using XRD. The $\mathrm{XRD}$ results is indicated that the particle size if nano $\mathrm{CuO}$ doped with lanthanum is much small as compared to that of $\mathrm{CuO}$ and decreases with lanthanum loading. From XRD results it is clear that as temperature increases, particle size also increases. The change in particle size cause large variation in the physical properties since $1 \mathrm{~nm}$ size change may introduce a considerable change in the number of surface atoms with lower consideration and broken exchange bonds. The UV absorption spectrum shows a red shift towards $389 \mathrm{~nm}$. The energy band gap for $\mathrm{La}$ doped $\mathrm{CuO}$ is calculated as $3.149 \mathrm{eV}$. The increasing red shift with decreasing particle size suggests that the defects responsible for the intra gap states are primarily surface defect. On doping band gap energy decreases. The red shift in the band gap energy and reduced electron hole recombination rate make the product material an ideal photo catalyst to harvest solar radiation for various applications. With decreasing particle size, a strong hybridization of the s-p states of the $\mathrm{CuO}$ host and the d states of the La impurity is likely to occur. The $\mathrm{La}$ doped $\mathrm{CuO}$ is highly effective in enhancing the photo catalytic degradation and could be used as a wide band gap semiconductor. 


\section{REFERENCES}

1. Yan L.,Zheng Y.B, Zhao F. Li S., Gao X., Xu B. Weiss P.S, Zhao Y, in: Chemistry and Physics of a single atomic layer in Strategies and challenges for functionalization of graphene and graphene-based materials, Chem.Soc.Rev. 41 (2012), 97-114.

2. Amelia, M.,Lincheneau C.,Silvi S., Credi., in: A. Electrochemical Properties of CdSe and CdTe quantum dots. Chem.Soc. Rev. 41 (2012), 5728-5743.

3. Zheng X.G., Xu C.N., Tomokiyo Y., Tanaka E., Yamada H. and Soejima Y., in: Observation of Charge Stripes in Cupric Oxide, Phys. Rev. Lett. 85 (2000), 5170.

4. Chang M.H., Liu H.-S.,Tai C.Y., in: Preparation of Copper Oxide nanoparticles and its application in nanofluid, Powder P.V.D.S. Technol. 207 (2011), 378.

5. Udani P.P.C., Gunawardana Lee H.C., Kim D.H, in: Steam reforming and oxidative steam reforming of methanol over $\mathrm{CuO}-\mathrm{CeO} 2$ catalysts, Int. J. Hydrogen Energy 34(2009), 7648.

6. Cao J.L., Shao G.S., Wang Y., Liu Y., Yuan Z.Y.,in: CuO catalysts supported on attapulgite clay for low-temperature CO oxidation, Catal. Commun. 9 (2008), 2555.

7. Chiang C.Y., Aroh K., Franson N., Satsangi V.R., Dass S., Ehrman S.,in: Copper oxide nanoparticle made by flame spray pyrolysis for photo electrochemical water splitting -Part II. Photo electrochemical study, Int. J. Hydrogen Energy 36 (2011), 15519.

8. [8]Langford J.I. Louer D., High-resolution powder diffraction studies of copper (II) oxide,J. Appl. Crystallogr. 24(1991)149.

9. Abaker M., Umar, A. Baskoutas S., Kim S.H., Hwang S.W., in: Structural and optical properties of $\mathrm{CuO}$ layered hexagonal discs synthesized by a low-temperature hydro-thermal process, J. Phys. D: Appl. Phys. 44 (2011), 155

10. Williamson G.K., Hall W.H, in: X-ray line broadening from filed Aluminum and wolfram, Acta Metall. 1 (1953), 22.

11. Moss M.A. Burrell T.S, Ellis G.J., in: Semiconductor Opto-Electronics, Butterworth \& Co. Ltd, 1973.

12. Honsi H.M., Fayek S.A., El-Sayed S.M., Roushdy M., in: Optical properties and DC electrical conductivity of $\mathrm{Ge}_{28-\mathrm{x}} \mathrm{Se}_{72} \mathrm{Sb}_{\mathrm{x}}$ thin films, Vacuum 81 (2006), 54.

13. Sawaby A., Selim M.S., Marzouk S.Y., Mostafa M.A. Hosny A, in: Structure, optical and electrochromic properties of NiO thin films", Physica B 4053412 (2010).

14. Ken Ohwade and Ginji Pujiswas in: Optical vibration of doped Lanthanum Copper Oxide compound, Applied Spectroscopy, 47, 3(1993), 296-99.

15. Golosovsky I.V, Gukasov A.G,Polyakov V.A, in : nonlinear anti- ferromagnetic structure with the propagation vector and the transition temperature of $130 \mathrm{~K}$ was found in monoclinic compound $\mathrm{La} 2 \mathrm{Cu} 2 \mathrm{O} 5$ by single crystal neutron diffraction, J Phys-Condensed Matter,11,36(1999)6959-67

16. Neeleshwar S, Chen C.L, Tsai C.B., Chen Y.Y., Chen C.C., Shyu S.G., Seehra M.S., in: dependent properties of CdSe quantum dots, Phys. Rev. B 71 (2005) 201,307

17. Nanda K K., Kruis F E., Hassan H., Phys.Rev.Lett. 89 (2002) 256103.

18. Gizhevskii B.A., Sukhorukov Y.P., Moskvin. A.S.,Loshkareva N.N., Mostovshchikova E.V., Ermakov A.E., Kozlov E.A., Uimin M.A., Gaviko V.S in : Anomalies in the Optical properties of Nanocrystalline Copper Oxides $\mathrm{CuO}$ and $\mathrm{Cu}_{2} \mathrm{O}$ near the fundamental absorption edge, JETP 102 (2006) 297. 
19. Sukhorukov Y.P., Gizhevskii B.A., Mostovshchikova E.V., Yermakov A.Y., Tugushev S.N., Kozlov E.A., in: Nano-crystalline Copper Oxide for selective solar energy absorbers, Tech. Phys.Lett. 32 (2006) 132.

20. Ovchinnikov S.G., Gizhevskii B.A, Sukhorukov Y.P., Ermakov A.E., Uimin M.A., Kozlov E.A., Kotov Y., Bagazeev A.A.V, in: Specific features of the electronic structure and optical spectra of nanoparticles with strong electron correlations, Phys. Solid State 49 (2007), 1116

21. Rehman S., Mumtaz A Hasanain S.K., in: Size effects on the Magnetic and Optical properties of $\mathrm{CuO}$ nanoparticles”, J. Nanopart. Res. 13 (2011), 2497.

22. Koffyberg F.P., Benko F.A., in: A Photoelectrochemical determination of the position of the conduction and valence band edges of p-type CuO , J. Appl. Phys. 53 (1982), 1173.

23. Neeleshwar S., C. Chen., Tsai L. C.B., Y. Chen., C.Y. Chen., C. Shyu S.G., Seehra M.S., in: Size-dependent properties of CdSe quantum dots, Phys. Rev. B 71 (2005), 201307.

24. Sukhorukov Y.P., Gizhevskii B.A., Mostovshchikova E.V., Yermakov A.Y., Tugushev S.N., Kozlov E.A.,in: Nano-crystalline Copper Oxide for selective solar energy absorbers, Tech. Phys.Lett. 32 (2006), 132.

25. Ovchinnikov S.G., Gizhevskii B.A., Sukhorukov Y.P., Ermakov A.E., Uimin A., Kozlov E.A., Kotov Y.,Bagazeev A.A.V.,in: Specific features of the Electronic structure and Optical spectra of nanoparticles with strong electron correlations, Phys. Solid State 49 (2007) 1116.

26. Koffyberg F.P., Benko F.A.,in: A photoelectrochemical determination of the position of the conduction and valence band edges of p-type CuO , J. Appl. Phys. 53 (1982), 1173.

27. Mushtaq A.D,Sang H, in: Synthesis character and Electrochemical properties of $\mathrm{CuO}$ nanostructure,J.Solid state Electrochemical 14(2010), 1719-26.

28. Kannaki k,Remesh PS, Hydrothermal synthesisi of $\mathrm{CuO}$ Nanostructure and their charactgerisation, In.J. of Scientific \& Engg.Res. 3,9 (2012), 1-4 